\title{
Impactos da agricultura no meio ambiente: principais tendências e desafios (Parte 2$)^{1}$
}

\author{
Mauricio Vaz Lobo Bittencourt ${ }^{*}$
}

\begin{abstract}
RESUMO - Dando prosseguimento ao artigo anterior, onde se enfatizou a importância das tendências e desafios dos impactos da agricultura sobre o ar e a água, o presente texto tem por finalidade discutir os impactos da agricultura sobre o solo e uso da terra. As expansões desordenadas da fronteira agrícola, incentivadas pelo aumento da população mundial, manejo inadequado e contaminação do solo, podem propiciar perspectivas nada otimistas na produção e produtividade agrícolas a nível global. A experiência dos países desenvolvidos pode servir de alerta aos países em desenvolvimento no que diz respeito aos impactos agroambientais, pois estes adotam padrões de intensidade de uso bastante similares àqueles. Diversas medidas estão sendo tomadas e estudadas de caráter regulatório, econômico e técnico pelos países desenvolvidos e em desenvolvimento para frear o ritmo de expansão dos diversos tipos de degradação ambiental, sendo que as técnicas de uso e revolvimento mínimo do solo podem ser boas alternativas para minimizar os problemas de degradação e mau uso da terra.
\end{abstract}

Palavras-chave: Agricultura. Impactos ambientais. Poluição. Tendências. Desafios.

\section{INTRODUÇÃO}

$\mathrm{Na}$ sequência ao artigo anterior, no qual se destacou a importância das tendências e desafios dos impactos da agricultura sobre o ar e a água, este texto tem por finalidade discutir os impactos da agricultura sobre o solo e uso da terra. De forma geral, seria importante mensurar tais impactos agroambientais, o que não é trivial. Um primeiro aspecto que chama a atenção é que existe muito debate a respeito de como tentar mensurar os efeitos biofísicos e econômicos de longo prazo, bem como o impacto espacial da agricultura.

Grande parte desta literatura preocupa-se com a degradação da terra, principalmente com a erosão. Apesar de a ênfase ser mais na questão da degradação física, existem alguns poucos estudos que tentam captar o custo econômico da degradação como uma proporção do PIB agrícola. Scherr (1999), por exemplo, estima anualmente as perdas devido à erosão do solo para vários países africanos. No entanto, tais estudos baseados em estimações agregadas da degradação física podem, por sua vez, serem viesados, sendo que políticas baseadas na degradação física talvez não representem os custos da economia como um todo. Um segundo

\footnotetext{
${ }^{1}$ Parte 1 do artigo publicado no volume 18, ano 5.

* Professor Adjunto do Departamento de Economia da Universidade Federal do Paraná (UFPR). Endereço eletrônico: mbittencourt@ufpr.br.
} 
aspecto diz respeito às mudanças na importância relativa de diferentes impactos ao longo do tempo. Por exemplo, têm se centrado a atenção em controlar fontes pontuais de poluição, sendo que outras fontes têm se tornado bastante importantes.

A experiência dos países desenvolvidos pode servir de alerta aos países em desenvolvimento no que diz respeito aos impactos agroambientais, pois estes adotam padrões de intensidade de uso bastante similares àqueles. Esta experiência pode servir para estimar os custos dos impactos agroambientais que a agricultura intensiva provoca que não são diretamente refletidos nos preços das commodities agrícolas, mas que podem servir para definição de opções e prioridades nas políticas e tecnologias a serem adotadas.

\section{PRINCIPAIS TENDÊNCIAS E DESAFIOS}

\subsection{USO DA TERRA}

Apesar de 11 \% (1,5 bilhões de ha) da terra do planeta (13,4 bilhões de ha) ser usada na produção agrícola (terra arável e terra com culturas permanentes), esta área representa apenas pouco mais de um terço $(36 \%)$ da terra estimada que pode ser utilizada na produção agrícola. Assim sendo, teoricamente cerca de 2,7 bilhões de ha poderiam ser utilizados na expansão da agricultura mundial, mas devido a muitas restrições em seu uso, pouco desta terra poderia ser cultivada.

Um dos principais desafios para o futuro é a pressão ambiental resultante do uso intensivo da terra, e não só pelas mudanças na cobertura e uso da terra.

Pelas Tabelas 1 e 2 notam-se 3 fontes de crescimento da produção: expansão no uso da terra arável, expansão na terra colhida e crescimento da produtividade. Apesar dos números ilustrados nesta tabela servirem apenas como indicadores grosseiros ${ }^{2}$, nota-se que cerca de $80 \%$ do aumento da produção agrícola em países em desenvolvimento virá da intensificação e da expansão no uso da terra arável remanescente $(67 \%$ do aumento na produtividade e $12 \%$ da maior intensidade de colheitas).

\footnotetext{
${ }^{2}$ Para uma discussão a respeito dos problemas acerca dos dados de uso de terra ver Alexandratos (1995).
} 
TABELA 1 - FONTES DE CRESCIMENTO DA PRODUÇÃO AGRÍCOLA MUNDIAL (\%)

\begin{tabular}{|c|c|c|c|c|c|c|c|c|}
\hline & \multicolumn{2}{|c|}{$\begin{array}{c}\text { Arable land } \\
\text { expansion } \\
\text { (1) } \\
\end{array}$} & \multicolumn{2}{|c|}{$\begin{array}{c}\text { Increases in } \\
\text { cropping intensity } \\
(2)\end{array}$} & \multicolumn{2}{|c|}{$\begin{array}{l}\text { Harvested land } \\
\text { expansion }(1+2)\end{array}$} & \multicolumn{2}{|c|}{ Yield increases } \\
\hline & 1961 & $1997 / 99$ & 1961 & $1997 / 99$ & 1961 & $1997 / 99$ & 1961 & $1997 / 99$ \\
\hline & -1999 & -2030 & -1999 & -2030 & -1999 & -2030 & -1999 & -2030 \\
\hline All Developing countries & 23 & 21 & 6 & 12 & 29 & 33 & 71 & 67 \\
\hline excl. China & 23 & 24 & 13 & 13 & 36 & 37 & 64 & 63 \\
\hline excl. China and India & 29 & 28 & 16 & 16 & 45 & 44 & 55 & 56 \\
\hline Sub-Saharan Africa & 35 & 27 & 31 & 12 & 66 & 39 & 34 & 61 \\
\hline $\begin{array}{l}\text { Near East/North Africa } \\
\text { Latin America and the }\end{array}$ & 14 & 13 & 14 & 19 & 28 & 32 & 72 & 68 \\
\hline Caribbean & 46 & 33 & -1 & 21 & 45 & 54 & 55 & 46 \\
\hline South Asia & 6 & 6 & 14 & 13 & 20 & 19 & 80 & 81 \\
\hline East Asia & 26 & 5 & -5 & 14 & 21 & 19 & 79 & 81 \\
\hline World & 15 & & 7 & & 22 & & 78 & \\
\hline All Developing countries & & & & & & & & \\
\hline Crop production - rainfed & & 25 & & 11 & & 36 & & 64 \\
\hline Crop production - irrigated & & 28 & & 15 & & 43 & & 57 \\
\hline
\end{tabular}
FONTE: FAO.

TABELA 2 - TERRA COM POTENCIAL DE PRODUÇÃO AGRÍCOLA

\begin{tabular}{|c|c|c|c|c|c|c|c|c|}
\hline & 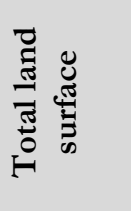 & 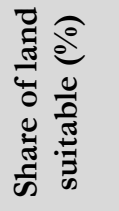 & 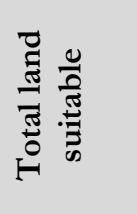 & $\begin{array}{l}\frac{0}{0} \\
\frac{\pi}{0} \\
0 \\
0 \\
0 \\
0 \\
0\end{array}$ & क & 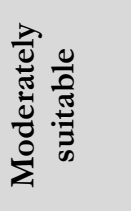 & 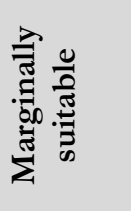 & $\begin{array}{l}\frac{0}{0} \\
\frac{\pi}{3} \\
0 \\
0 \\
0 \\
\text { Z }\end{array}$ \\
\hline Developing countries & 7302 & 38 & 2782 & 1109 & 1001 & 400 & 273 & 4520 \\
\hline Sub-Saharan Africa & 2287 & 45 & 1031 & 421 & 352 & 156 & 103 & 1256 \\
\hline Near East/North Africa & 1158 & 9 & 99 & 4 & 22 & 41 & 32 & 1059 \\
\hline Latin America and the Caribbean & 2035 & 52 & 1066 & 421 & 431 & 133 & 80 & 969 \\
\hline South Asia & 421 & 52 & 220 & 116 & 77 & 17 & 10 & 202 \\
\hline East Asia & 1401 & 26 & 366 & 146 & 119 & 53 & 48 & 1035 \\
\hline Industrial countries & 3248 & 27 & 874 & 155 & 313 & 232 & 174 & 2374 \\
\hline Transition countries & 2305 & 22 & 497 & 67 & 182 & 159 & 88 & 1808 \\
\hline World $^{3}$ & 13400 & 31 & 4188 & 1348 & 1509 & 794 & 537 & 9211 \\
\hline
\end{tabular}

FONTE: FAO (2003).

Deste modo, a relação de custo/benefício agroambiental continuará sendo baseada nos incrementos no uso de terra para a agricultura que, por sua vez, reflete o uso de cultivares melhorada, maior uso de insumos como adubos e rações animais, juntamente com melhores práticas no cultivo, e melhor gerenciamento no uso de pesticidas e irrigação. No entanto, existe uma preocupação contínua com respeito à degradação de áreas frágeis e ricas em biodiversidade. Os benefícios positivos destas mudanças estão na redução da erosão e o menor aumento na poluição por fertilizantes e pesticidas. No entanto, as perspectivas são pessimistas quanto aos níveis de nitrato nos lençois freáticos devido à inadequada gestão no

\footnotetext{
${ }^{3}$ Inclui alguns países não cobertos por este estudo.
} 
uso de fertilizantes, ocasionando posterior perda de terras e produtividade devido à salinização, e o crescimento da poluição do ar e água devido à produção animal.

Os principais problemas agroambientais estão subdivididos em 2 grupos:

1) Aqueles de escala global, tal como o aumento na produção de $\mathrm{CO}_{2}$ através da perda de área florestal, e óxido nitroso $\left(\mathrm{N}_{2} \mathrm{O}\right)$ oriundo das lavouras (HOUGHTON et al., 1995; MOSIER \& KROEZE, 1998).

2) Os de menor escala, ou locais, nos principais países e continentes, que até o presente não tem grandes impactos a nível global. Por exemplo, salinização de áreas irrigadas, e o aumento na contaminação de águas superficiais e subterrâneas com resíduos de fertilizantes nitrogenados.

Apesar destes problemas serem conhecidos desde o início dos anos 1970, como consequência do uso intensivo do solo pela agricultura, os mesmos vêm ganhando importância cada vez maior em países em desenvolvimento nos anos recentes, o que pode se tornar ainda mais importante nos próximos anos, a menos que tenhamos uma mudança na trajetória das políticas e da tecnologia vigentes nestes países.

A maior parte dos impactos negativos da agricultura sobre o meio ambiente poderia ser reduzida ou prevenida por uma apropriada miscelânea de políticas e mudanças tecnológicas (ALEXANDRATOS, 1995; PRETTY, 1995; CONWAY, 1997). Com as recentes, e crescentes, pressões para que a agricultura se torne menos maléfica ao meio ambiente, estas pressões "forçam" os países a reduzirem as pressões sobre os preços das commodities e subsídios no uso de insumos, o que possibilita a redução de intervenções políticas que só tendem a piorar os impactos agroambientais negativos, através da integração das questões ambientais nas formulações de políticas agrícolas.

\subsection{IMPACTOS NO SOLO}

Um dos mais importantes problemas ambientais em anos recentes têm sido a remoção da cobertura do solo, particularmente o desmatamento e o uso intensivo da terra, com sérios impactos na degradação do solo. O que vem acontecendo é uma redução na conversão de áreas florestais em lavouras ou pastagens, e aumento em áreas protegidas. No entanto, projeções e tendências a respeito de degradação do solo permanecem dividindo opiniões, pois as bases empíricas para tais projeções são ainda muito fracas. 


\subsubsection{Alteração na cobertura do solo}

Como a maior parte das alterações na cobertura do solo vem do desmatamento, este tipo de alteração tende a se reduzir ao longo do tempo, apesar de ainda persistir por algum tempo nos trópicos. $\mathrm{Na}$ América Latina os países têm procurado remover algumas políticas que incentivavam grandes donos de terras a desmatar e criar amplas áreas de pastagens. No entanto esta prática continua ocorrendo dentre os pequenos proprietários de terras. $\mathrm{Na}$ África Ocidental, praticamente não existe floresta natural, sendo que $80 \%$ da população vive em áreas rurais e cresce de 2,5 a 3\% ao ano.

Como existe uma redução na expansão no uso de terras para agricultura, conforme Tabela 3, existem 3 pontos de preocupação. Primeiro, a frequência e intensidade no uso de terras íngremes cobertas primariamente com florestas deve aumentar. Segundo, retirada de água e uso de áreas de banhados e mangues irão reduzir a biodiversidade e a reprodução de peixes, aumentando a produção de $\mathrm{CO}_{2}$, mas reduzindo a emissão de metano. Terceiro, terras de alta qualidade continuarão a ser cedidas para o desenvolvimento industrial e urbano.

TABELA 3 - ÁREA AGRICULTURÁVEL TOTAL NO PASSADO E PROJETADO PARA O FUTURO

\begin{tabular}{|c|c|c|c|c|c|c|c|c|c|c|c|c|}
\hline & \multicolumn{6}{|c|}{ Arable land in use } & \multicolumn{2}{|c|}{$\begin{array}{l}\text { Annual } \\
\text { growth }\end{array}$} & \multicolumn{2}{|c|}{$\begin{array}{c}\text { Land in use } \\
\text { as } \% \text { of } \\
\text { potential }\end{array}$} & \multicolumn{2}{|c|}{ Balance } \\
\hline & $\begin{array}{c}1961 \\
/ 63\end{array}$ & $\begin{array}{c}1979 \\
/ 81\end{array}$ & $\begin{array}{c}1997 \\
/ 99\end{array}$ & $\begin{array}{l}1997 \\
/ 99 \\
\text { adj. }\end{array}$ & $\begin{array}{l}2015 \\
\text { (mi }\end{array}$ & $\begin{array}{l}2030 \\
\text { on ha) }\end{array}$ & $\begin{array}{c}1961- \\
1999\end{array}$ & $\begin{array}{c}1997 / \\
99- \\
2030 \\
\text { o p.a.) }\end{array}$ & $\begin{array}{c}1997 \\
199\end{array}$ & $\begin{array}{r}2030 \\
(\%)\end{array}$ & $\begin{array}{l}1997 \\
/ 99 \\
\quad \text { (mil }\end{array}$ & $\begin{array}{l}2030 \\
\text { Ion ha) }\end{array}$ \\
\hline $\begin{array}{l}\text { Sub-Saharan } \\
\text { Africa }\end{array}$ & 119 & 138 & 156 & 228 & 262 & 288 & 0.77 & 0.72 & 22 & 28 & 803 & 743 \\
\hline $\begin{array}{l}\text { Near East/ } \\
\text { North Africa } \\
\text { Latin America } \\
\text { and the }\end{array}$ & 86 & 91 & 100 & 86 & 89 & 93 & 0.42 & 0.23 & 87 & 94 & 13 & 6 \\
\hline Caribbean & 104 & 138 & 159 & 203 & 223 & 244 & 1.22 & 0.57 & 19 & 23 & 863 & 822 \\
\hline South Asia & 191 & 202 & 205 & 207 & 210 & 216 & 0.17 & 0.13 & 94 & 98 & 13 & 4 \\
\hline excl. India & 29 & 34 & 35 & 37 & 38 & 39 & 0.37 & 0.12 & 162 & 168 & -14 & -16 \\
\hline East Asia & 176 & 182 & 227 & 232 & 233 & 237 & 0.89 & 0.06 & 63 & 65 & 134 & 129 \\
\hline excl. China & 72 & 82 & 93 & 98 & 105 & 112 & 0.82 & 0.43 & 52 & 60 & 89 & 75 \\
\hline $\begin{array}{l}\text { Developing } \\
\text { countries }\end{array}$ & 676 & 751 & 848 & 956 & 1017 & 1076 & 0.68 & 0.37 & 34 & 39 & 1826 & 1706 \\
\hline $\begin{array}{l}\text { excl. China } \\
\text { excl. China and }\end{array}$ & 572 & 652 & 713 & 822 & 889 & 951 & 0.63 & 0.46 & 32 & 37 & 1781 & 1652 \\
\hline $\begin{array}{l}\text { India } \\
\text { Industrial }\end{array}$ & 410 & 483 & 543 & 652 & 717 & 774 & 0.81 & 0.54 & 27 & 32 & 1755 & 1633 \\
\hline $\begin{array}{l}\text { countries } \\
\text { Transition }\end{array}$ & 379 & 395 & 387 & & & & 0.07 & & 44 & & 487 & \\
\hline countries & 291 & 280 & 265 & & & & -0.19 & & 53 & & 232 & \\
\hline World & 1351 & 1432 & 1506 & & & & 0.34 & & 36 & & 2682 & \\
\hline
\end{tabular}

FONTE: Colunas (1) a (3): FAOSTAT, Novembro de 2001. 
Áreas íngremes são muito propensas a sofrerem de problemas de erosão quando utilizadas pela agricultura e apresentam inclinação entre 10 e $30 \%$, não se utilizam de técnicas apropriadas de cultivo e ocorrem fortes precipitações. No sudeste da Ásia, a pressão pelo uso da terra devido ao aumento populacional tem levado ao aumento no uso de terras íngremes, principalmente para plantio de milho, o que tem levado a diversos problemas de erosão nestas regiões (HUIZING \& BRONSVELD, 1991). Dois problemas para o futuro podem ser destacados. Primeiro, mais florestas poderão ser derrubadas para dar lugar a lavouras, resultando em mais erosão e perda de biodiversidade. Segundo, com aumento na intensidade de uso das áreas íngremes exploradas para cultivo agrícola, aumenta a degradação do solo principalmente pela erosão do solo ${ }^{4}$ (SHAXSON, 1998).

À medida que a população cresce, importante proporção de terra é perdida para o desenvolvimento industrial e urbano, estradas e reservas. Mas estas perdas parecem ser inevitáveis pelo baixo retorno do capital e trabalho agrícolas comparativamente aos usos nãoagrícolas. Como estas perdas são irreversíveis, as implicações para questões de segurança alimentar são bastante sérias. A magnitude em que estas perdas vão ocorrer e a proporção de terras de boa qualidade perdidas no futuro é incerta, no entanto as perdas devem ser substanciais. De acordo com Ash e Edmunds (1998), as perdas na China entre 1985 e 1995 foram superiores a 2 milhões de ha, sendo que a taxa de perda para a construção industrial vem aumentando desde 1980.

\subsubsection{Degradação do solo}

De acordo com alguns analistas a degradação do solo é uma das maiores ameaças à segurança alimentar, tem contribuído para anular os ganhos de produtividade do passado, e vem piorando ao longo do tempo (PIMENTEL et al., 1995; BREMEN, GROOT e VAN KEULEN, 2001).

O mais completo estudo a este respeito é o chamado GLASOD - Global Assessment of Human-Induced Soil Degradation (Tabela 4).

Os resultados demonstrados na Tabela 4 são incertos quanto aos impactos na produtividade e na classificação do grau de degradação. De acordo com FAO, UNDP, UNEP (1994), cerca de 30 a 40\% da área agrícola no Sul da Ásia tem algum grau de degradação, sendo que a erosão é o problema mais comum. No entanto, existem diversos problemas de

\footnotetext{
${ }^{4}$ Em países como Butão e Nepal este problemas são ainda mais críticos, pois toda e qualquer expansão de terras ocorre em áreas íngremes, devido ao esgotamento das terras planas nestes países.
} 
mensuração com a degradação do solo, dificultando a obtenção de informações e estimações precisas destas perdas.

TABELA 4 - AVALIAÇÃO GLOBAL DA DEGRADAÇÃO DO SOLO INDUZIDA PELO HOMEM $(\text { GLASOD })^{5}$

\begin{tabular}{lrrr}
\hline \multicolumn{1}{c}{ Region } & $\begin{array}{c}\text { Total land affected } \\
\text { (million ha) }\end{array}$ & \multicolumn{2}{c}{ Percentage of region degraded } \\
\hline & 494 & Moderate & Strong and extreme \\
Africa & 747 & 39 & 26 \\
Asia & 103 & 46 & 15 \\
Australasia & 243 & 4 & 2 \\
South America & 63 & 47 & 10 \\
Central America & 219 & 56 & 41 \\
Europe & 96 & 66 & 6 \\
North America & 1964 & 81 & 1 \\
Total & & 46 & 16 \\
\hline
\end{tabular}

FONTE- Oldeman, Hakkeling and Sombroek (1991).

Como a degradação é um processo lento e quase invisível, os aumentos de produção devido ao maior uso de insumos acabam por mascarar o impacto da degradação sobre a produtividade. Estimações dos custos econômicos da degradação são igualmente problemáticos, pois os impactos poderiam ocorrer não só nos níveis de produção, mas na qualidade dos bens produzidos, na estabilidade da produção e nos custos de produção (LIPPER, 2000). Adicionalmente, é necessário separar o efeito dos diferentes fatores envolvidos na produtividade total dos fatores (MURGAI, ALI \& BYERLEE, 2001). Existem também impactos externos à atividade agrícola, como salinização de córregos e mananciais, aumento no custo de armazenamento de água, e incidência de enchentes que são ainda mais sérios que os impactos nas atividades agrícolas.

De acordo com Crosson (1997), as recentes taxas de degradação do solo, particularmente a erosão, têm tido apenas um pequeno impacto na produtividade. Quase todas as áreas cultivadas de arroz no sul e sudeste da China são classificadas como afetadas por altos níveis de erosão. Mas Lindert (2000) sugere que tem tido muito pouca deterioração do solo na China desde os anos 1950, sendo que a erosão é muito baixa nos campos de arroz (NORSE et al., 2001). Ou seja, os aumentos de produção podem ter mascarado as possíveis perdas de produtividade.

Assim, temos:

\footnotetext{
${ }^{5}$ Glasod define 4 níveis de degradação: leve: com algum grau de queda na produtividade; moderada: grande redução na produtividade; forte: não recuperável ao nível de cultivo; extrema: sem recuperação com a tecnologia disponível.
} 
1) As estimativas locais e globais são bastante conflitantes devido às dificuldades de mensuração dos impactos da degradação do solo. Existem vários locais específicos onde a degradação já é séria e pode ficar mais séria (SCHERR e YADAV, 1996). Estas áreas incluem alguns dos países em desenvolvimento com áreas bastante férteis as quais desempenham papel muito importante na segurança alimentar (Tabela 5).

2) As áreas mais visíveis de degradação tendem a serem as terras marginais, enquanto as maiores produções tendem a ocorrer nas terras mais favoráveis, particularmente em áreas irrigadas (NORSE, 1988).

3) Existem fatores que podem contribuir para reduzir os efeitos da degradação do solo no futuro, como medidas diretas de prevenção e reversão (BRANCA, 2001), e medidas indiretas como o uso de técnicas de irrigação, inclusive evitando a salinização.

TABELA 5 - REGIÕES MAIS EVIDENTES DA DEGRADAÇÃO DO SOLO

\begin{tabular}{|c|c|c|}
\hline Region & Salinization & Erosion \\
\hline South and West Asia & $\begin{array}{r}\text { Indus, Tigris and Euphrates } \\
\text { river basins }\end{array}$ & Foothills of the Himalayas \\
\hline East and Southeast Asia & $\begin{array}{l}\text { Northeast Thailand and } \\
\text { North China Plain }\end{array}$ & $\begin{array}{r}\text { Unterraced slopes of China and Southeast } \\
\text { Asia }\end{array}$ \\
\hline Africa & Nile Delta & $\begin{array}{l}\text { Southeast Nigeria, the Sahel, mechanized } \\
\text { farming areas of North and West Africa }\end{array}$ \\
\hline $\begin{array}{l}\text { Latin America and the } \\
\text { Caribbean }\end{array}$ & $\begin{array}{r}\text { Northern Mexico, Andean } \\
\text { highlands }\end{array}$ & $\begin{array}{l}\text { Slopes of Central America, the semi-arid } \\
\text { Andean Valley and the cerrados of Brazil }\end{array}$ \\
\hline
\end{tabular}
FONTE: Scherr e Yadav (1996).

Na Tabela 6 pode-se notar que cerca de $12,5 \%$ da área projetada para ser colhida em 2030 nos países da América Latina deverão vir de terras irrigadas ${ }^{6}$, as quais são geralmente terras planas com pouca erosão, mas com certo risco de salinização, principalmente em áreas mais áridas (NORSE et al., 2001). Outro aspecto a ser enfatizado é a possibilidade de crescimento de áreas sem irrigação, mas com o uso do plantio direto, que propicia ganhos como redução de erosão, da perda de nutrientes advindos das plantas, alta infiltração de águas pluviais e melhor capacidade do solo em reter umidade, ou seja, o plantio direto melhora as condições físicas, químicas e biológicas do solo, elevando o nível de matéria orgânica no solo.

Adicionalmente, o uso mais eficiente de fertilizantes tende a aumentar, o que poderá trazer maiores benefícios ao solo em termos de níveis de nutrientes e de matéria orgânica.

\footnotetext{
${ }^{6}$ Esta proporção chega a $32 \%$ se considerarmos os países em desenvolvimento como um todo, conforme a Tabela 6.
} 
Com isso, a erosão tenderá a se reduzir com o maior desenvolvimento de raízes e plantas, com a maior cobertura de fosfato e potássio.

TABELA 6 - ÁREA AGRICULTÁVEL EM USO, INTENSIDADE DE CULTIVO E ÁREA COLHIDA

\begin{tabular}{|c|c|c|c|c|c|c|c|c|c|c|}
\hline & & Total & and $\mathrm{i}$ & use & & fed $x$ & & Irri & ated & \\
\hline & & A & $\mathrm{Cl}$ & $\mathbf{H}$ & A & $\mathrm{Cl}$ & $\mathbf{H}$ & A & $\mathrm{Cl}$ & $\mathbf{H}$ \\
\hline & $1997 / 9$ & & & & & & & & & \\
\hline Sub-Saharan Africa & 9 & 228 & 68 & 154 & 223 & 67 & 150 & 5 & 86 & 4.5 \\
\hline & 2030 & 288 & 76 & 217 & 281 & 75 & 210 & 7 & 102 & 7 \\
\hline & $1997 / 9$ & & & & & & & & & \\
\hline Near East/ North Africa & 9 & 86 & 81 & 70 & 60 & 72 & 43 & 26 & 102 & 27 \\
\hline & 2030 & 93 & 90 & 83 & 60 & 78 & 46 & 33 & 112 & 37 \\
\hline & $1997 / 9$ & & & & & & & & & \\
\hline Latin America and the Caribbean & 9 & 203 & 63 & 127 & 185 & 60 & 111 & 18 & 86 & 16 \\
\hline & 2030 & 244 & 71 & 172 & 222 & 68 & 150 & 22 & 100 & 22 \\
\hline & $1997 / 9$ & & & & & & & & & \\
\hline South Asia & 9 & 207 & 111 & 230 & 126 & 103 & 131 & 81 & 124 & 100 \\
\hline & 2030 & 216 & 121 & 262 & 121 & 109 & 131 & 95 & 137 & 131 \\
\hline & $1997 / 9$ & & & & & & & & & \\
\hline East Asia & 9 & 232 & 130 & 303 & 161 & 120 & 193 & 71 & 154 & 110 \\
\hline & 2030 & 237 & 139 & 328 & 151 & 122 & 184 & 85 & 169 & 144 \\
\hline & $1997 / 9$ & & & & & & & & & \\
\hline All above & 9 & 956 & 93 & 885 & 754 & 83 & 628 & 202 & 127 & 257 \\
\hline & 2030 & 1076 & 99 & 1063 & 834 & 87 & 722 & 242 & 141 & 341 \\
\hline & $1997 / 9$ & & & & & & & & & \\
\hline excl. China & 9 & 822 & 83 & 679 & 672 & 76 & 508 & 150 & 114 & 171 \\
\hline & 2030 & 951 & 90 & 853 & 769 & 81 & 622 & 182 & 127 & 230 \\
\hline & $1997 / 9$ & & & & & & & & & \\
\hline Excl. China/India & 9 & 652 & 75 & 489 & 559 & 70 & 392 & 93 & 105 & 97 \\
\hline & 2030 & 774 & 83 & 641 & 662 & 77 & 507 & 112 & 119 & 134 \\
\hline
\end{tabular}

FONTE: FAO

NOTA: A= área agricultável em milhões de ha; $\mathrm{CI}=$ intensidade de cultivo em \%; $\mathrm{H}=$ área colhida em milhões de ha.

Ou seja, as perdas globais pela degradação do solo poderão ser pequenas, mas as perdas em certas áreas e regiões poderão ser significativas. No entanto, as perdas de produtividade do solo pela sua degradação podem ser bem mais sérias se os ganhos com a expansão do uso de técnicas como o plantio direto e conservação do solo ficarem abaixo do previsto, e o crescimento das colheitas caírem gradativamente.

Acredita-se que algumas áreas mais frágeis e propensas à degradação serão abandonadas no futuro, o que poderá não resultar em pressões por áreas a serem desmatadas, devido à reestruturação da agricultura e às altas taxas de urbanização e migração rural-urbana projetadas para o futuro ${ }^{7}$. Isto já vem acontecendo em alguns países em desenvolvimento, como a China, na qual a urbanização está sendo acompanhada por mudanças em fontes de renda alternativas.

\footnotetext{
${ }^{7}$ Esta migração ocorreu nos anos 1950 e 60 na Europa, levando a uma redução na utilização de terras íngremes e outras áreas marginais, as quais se transformaram em áreas florestais, por exemplo, conforme Baldock et al. (1996).
} 
A desertificação também vem sendo um importante tipo de degradação do solo, causado muitas vezes por excesso de uso do solo, manejo inadequado do mesmo, e sistemas de cultivo e colheita inapropriados. No caso do futuro, pode-se pensar em algumas possibilidades para evitar este problema, tais como: redução no uso de produção extensiva de rebanhos em áreas áridas e evitar a superlotação de animais nas pastagens; uso de sistemas de irrigação, técnicas de armazenamento de água, e medidas para evitar a salinização do solo; uso da técnica de plantio direto; melhores cultivares de cobertura do solo, mais resistentes à falta de água; maiores esforços dos governos de modo a recuperarem terras em processo de desertificação.

\section{CONSIDERAÇÕES FINAIS}

Ficou demonstrado que muitos dos negativos impactos ambientais da agricultura são consequência da maior intensificação global no uso da terra ao longo do tempo. As políticas, sucessos e insucessos das tecnologias adotadas pelos países desenvolvidos podem ser de grande ajuda para os países em desenvolvimento que estão se deparando com os problemas ambientais com o uso intensificado da agricultura. Muitas das políticas e ações regulatórias e tecnológicas são conhecidas, e que se adotadas poderão resultar em um cenário agroambiental mais favorável no futuro.

As técnicas de uso e revolvimento mínimo do solo são uma alternativa para vários produtores em diversos países. Os governos devem direcionar suas políticas e propiciar condições para que tais técnicas como, por exemplo, o plantio direto, possam reduzir os impactos negativos da agricultura no meio ambiente. Grandes esforços de investimentos e desenvolvimentos serão necessários para que estas técnicas sejam criadas e disseminadas para os sistemas produtivos em vários países e regiões.

\section{REFERÊNCIAS}

ALEXANDRATOS, N. World agriculture: towards 2010. ed. 1995. An FAO study. Chichester, UK, John Wiley and Rome, FAO.

ASH, R. AND EDMUNDS R. China's land resources, environment and agricultural production. The China Quarterly. set./1998.

BREMEN, H., GROOT, J. AND VAN KEULEN, H. 2001. Resource limitations in Sahelian agriculture. Global Environmental Change, 11: 59-68.

CONWAY, G. The doubly green revolution: food for all in the 21st century. London: Penguin Books. 1997. 
FAO. The use of saline waters for crop production. FAO Irrigation and Drainage Paper No. 48. Rome. 1993.

HOUGHTON, J., MEIRA FILHO, L., CALLANDER, B., HARRIS, N., KATTENBERG, A. AND K. MASKELL. Radiative forcing of climate change and an evaluation of the IPCC IS92 emission scenarios. eds. 1995. Cambridge, UK, Cambridge University Press.

HUIZING, H. AND BRONSVELD, K. The use of geographical information systems and remote sensing for evaluating the sustainability of land-use systems. In Evaluation for sustainable land management in the developing world. IBSRAM Proceedings. Bangkok, v.2, n.12, p. 545-562. 1991.

LIPPER, L. Dirt poor: poverty, farmers and soil resource investment. Rome, 2000. FAO. (mimeo)

MOSIER, A. AND KROEZE, C. A new approach to estimate emissions of nitrous oxide from agriculture and its implications for the global change N2O budget. IGBP Global Change Newsletter, 34, p. 8-13. jun./1998.

OLDEMAN, L., HAKKELING, R. AND SOMBROEK, W. World map of the status of human-induced soil degradation, second revised ed. Wageningen, the Netherlands, ISRIC and Nairobi, UNEP. 1991.

PIMENTEL, D., HARVEY, C., RESOSUDARMO, P., SINCLAIR, K., KURZ, D., MCNAIR, M., CHRIST, L., SHPRITZ, L., FITTON, L., SAFFOURI, R. AND BLAIR, R. Environmental and economic costs of soil erosion and conservation benefits. Science, 267, p. 1117-1123. 1995

PRETTY, J. Regenerating agriculture: policies and practice for sustainability and selfreliance. London: Earthscan. 1995.

SCHERR, S. Soil degradation: a threat to developing country food security by 2015? Food, Agriculture and the Environment Discussion Paper. Washington, DC, IFPRI. n. 27. 1999.

SHAXSON, F. New concepts and approaches to land management in the tropics with emphasis on steep lands. Rome, FAO. 1998. 
